\title{
OBSERVATIONAL CONSTRAINTS ON MODE EXCITATION IN $\delta$-SCUTI STARS IN OPEN CLUSTERS
}

\author{
S. FRANDSEN AND H. KJELDSEN \\ Institute of Physics and Astronomy, Århus University, Denmark
}

\section{SEISMOLOGY WITH $\delta$-SCUTI STARS}

The special opportunities offered by $\delta$-Scuti stars are the following: - They regularly oscillate in several modes, and with high precision observations one has measured of the order 5 modes (Michel and Baglin 1991).

- The excitation is a very delicate and therefore a very sensitive measure of the internal structure.

- They are found in open clusters which constitute a very rich scenario for tests of the theory of stellar structure and evolution. This makes it feasible to observe several stars simultaneously and gain more results than for single stars.

- The brightness is moderately high and still they live long enough to be present in a variety of clusters and in the field as well.

\section{EXCITATION PROBLEMS}

Some of the questions we would like to see answers to are the following: - When do modes become excited? What is the relation between excitation and rotation and how does the chemical composition influence excitation?

- If modes are excited, why exactly the few modes we see among the large set of possible modes?

- Why do we see amplitude changes in some stars?

\section{THE OLD OPEN CLUSTERS}

In this paper we display results based on an analysis of 5 old open clusters. We have used high precision time series CCD photometry to observe a large number of stars of which many of the brighter are situated in the instability strip. The clusters included are: NGC 1513, NGC 2423, NGC 2660, NGC 6134 and NGC 6802 . The technique has been gradually refined during the project and we have a fairly inhomogenous material for the various clusters. The main achievement can be described in two paragraphs:

a. The number (and probably type) of variables found seem to vary from cluster to cluster. This may serve as a probe of the internal constitution of the stars (needs to be confirmed).

b. In one case (NGC 6134) we do have data enough to make an individual analysis of the variables. As shown elsewhere this leads to a precise knowledge of the modes excited, except that proper models of the stars do not exist yet. 


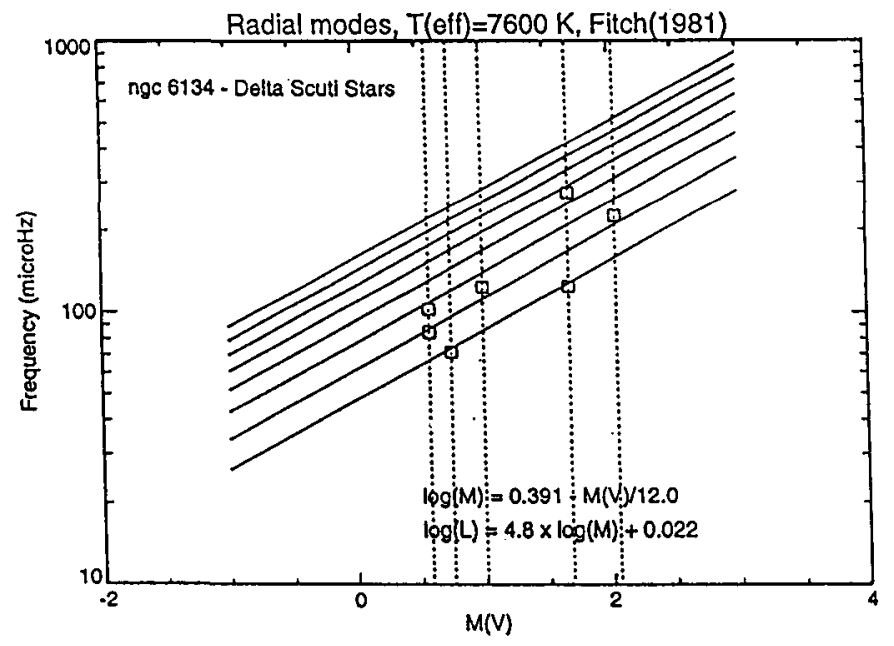

FIGURE I Figure demonstrating how modes may be identified when observing stars in a cluster. There is a freedom to move the points in the horizontal direction to fit the bottom line which is the fundamental frequency at a given luminosity.

\section{TECHNIQUES FOR MODE IDENTIFICATION}

The mode spectrum for an evolved $\delta$-Scuti star is very dense and very complicated (Dziembowski and Krolikowska 1990). During evolution the p-modes switch character and change into g-modes, which complicates the analysis and the identification very much. The tools suggested to be used for mode identification are:

- First of all long time series with few missing windows to resolve the frequency spectrum and to allow precise frequency determination.

- Time series of the colour of a variable to study phase shifts, which depend on the $l$-value of the mode.

- Doppler imaging of the surface in fast rotators to identify the surface pattern characteristics of modes.

- Differential analysis of variables in cluster (see next)

\section{A NEW SCHEME FOR MODE IDENTIFICATION}

1. In a cluster we can fix accurately the positions of the stars in a colourluminosity diagram except for a zero point on each axis.

2. For simplicity we assume for NGC 6134 that the 5 stars have the same effective temperature, which is nearly the case. We also assume the simple models computed by Fitch (1981) are adequate. Some of the stars oscillate in the fundamental mode. Fitting these frequencies to the theoretical we can determine the zero point for the luminosity precisely (see Figure I). Now all overtones can be identified by reading them off the figure, f.ex. as being a $p_{5}$ mode. With a more numerous set of variables the chances are that also a metal content and an age can be determined due to the constraints imposed by a large set of fre- 


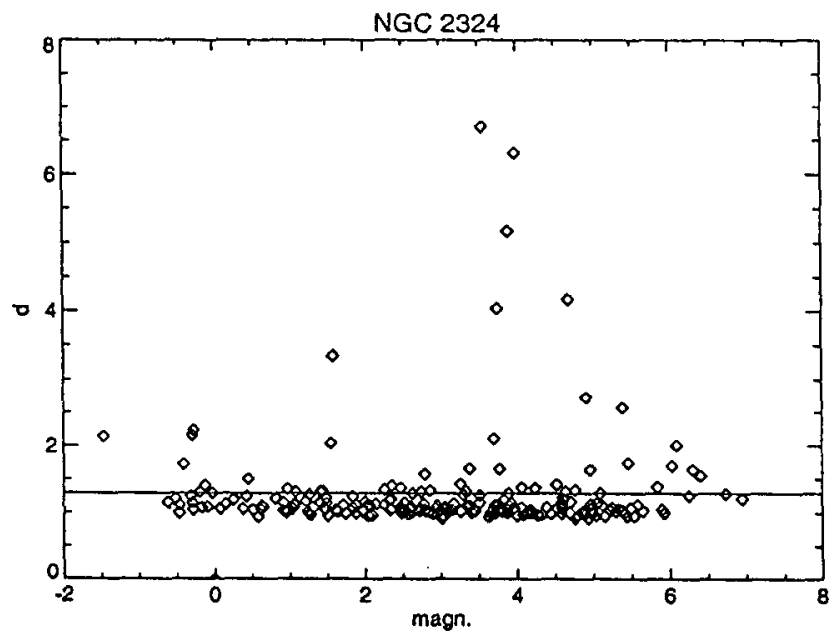

FIGURE II NGC 2324. There are a small number of variables, but the time string is too short to allow an analysis of the variables detected. Note once more the large number of constant stars.

quencies. Also approximate values for these parameters are known from classical techniques used in clusters, which provide a first good estimate.

\section{THE SEARCH FOR VARIABILITY}

We search for variables by calculating a quantity $d$. This is the ratio between the r.m.s. scatter around the mean of the time series of magnitudes for a given star divided by another r.m.s. scatter obtained by taking differences between successive measurements.

To search for variables we plot $d$ against magnitude and then take a note of all stars above a certain value of $d$ depending on the number of frames available for a given cluster. In each diagram the brighter stars have been through a selection where stars affected by CCD defects or other disturbing sources have been rejected. An example is given as Figure II. The fraction of variables detected is very small compared to expected ( $30 \%$ for field stars) in most clusters.

\section{REFERENCES}

Breger, M. 1979, Publ. Ast. Soc. Pac. 91, 5

Dziembowski, W. and Królikowska, M. 1990, Acta Astron. 40, 19

Fitch, W.S. 1981, Astroph. J. 249, 218

Michel, E. and Baglin, A. 1991, Adv. Space Res. 11, 70 